\title{
Aspekte der Vertikalverteilung von Spinnen (Araneae) an Kiefernstämmen
}

\author{
Daniel BRAUN
}

\begin{abstract}
Aspects of vertical distribution of spiders (Araneae) on pine trunks. From May to October 1991, spiders on pine trunks in a moorish pine forest in the Federsee nature reserve in Upper Swabia were caught with arboreal photoeclectors. To register vertical differences of intensity of activity, the eclectors were attached at different heights above the ground, one eclector on each trunk.

Abiotic parameters (temperature, evaporation), the structure of the bark and its epiphytes were recorded. 108 species from 19 families were found. Mostfrequent were the families Linyphiidae, Theridiidae and Anyphaenidae. The most abundant species was Entelecara penicillata. Intensity of activity, ecotype, dominance structure, dominance identity, diversity and evenness were used as parameters to investigate vertical changes in fauna structure. The investigations showed that the lowest trunk-region is frequently used by spiders normally living in the field layer or the litter. These species, however, hardly ever climb up to higher regions. On the other hand, the eclector faunas of the middle and especially of the highest parts of the trunks near the canopies are heavily influenced by the canopy fauna. Several epitruncal species also show preferences for these different trunk-regions. Ecological and abiotic parameters show that the upper trunk-region is a much more extreme habitat than the lower one and the fauna structures are in accordance with this.
\end{abstract}

Key words: Araneae, pine trunks, arboreal photoeclectors, vertical distribution.

\section{EINLEITUNG}

Etwa die Hälfte der einheimischen Spinnenarten kann als fakultativ oder obligat silvicol angesehen werden. Die meisten davon zählen zu den Bewohnern der Streu- und Krautschicht, wohingegen besonders die Stammregion vergleichsweise recht spärlich besiedelt ist. Als exclusive Rindenspinnen sieht WUNDERLICH (1982) lediglich 1,2\% der mitteleuropäischen Arten an. Jedoch werden die Stämme von Vertretern anderer Strata häufig fakultativ genutzt.

Die verschiedenen Stratocoenosen sind im allgemeinen nicht streng voneinander separiert, so daß es zu Überlappungen und Vermischungen 
kommen kann. Nach LUZCAK (1959) ist die Streuschicht nur während der Vegetationsperiode in ihrer Besiedlung deutlich von den anderen Schichten getrennt. Mit Einbruch der kalten Jahreszeitsuchen viele Bewohner der höheren Strata den Boden auf, um das dort zur Überwinterung günstigere Mikroklima zu nutzen (ENGELHARDT 1958, LUCZAK 1959). Zu weiteren Wanderungen zwischen den Strata kommt es in der Kopulations- und Postkopulationszeit, bei der Ausbreitung der Juvenilen oder auf der Nahrungssuche (VITÉ 1953). Ferner gibt es Arten, die in Abhängigkeit von der Vegetationsstruktur sowohl Krautschicht als auch Büsche und Bäume besiedeln können (LUCZAK 1959, 1963, 1966). Hierbei ist zu beachten, daß besonders für netzbauende Spinnen Charakteristika der Pflanzen wie Wuchsform, Strukturierung und Verzweigung meist wesentlich wichtiger sind als die Lage der Vegetationsschicht, in der diese Strukturen angetroffen werden (LUCZAK 1959, SCHEIDLER 1990).

Daneben findet sich aber eine Reihe von Arten, die sehr enge Stratotopbindungen zeigen.

\section{MATERIAL UND METHODEN}

\section{Untersuchungsgebiet}

Die Untersuchungen wurden im "Banngebiet Staudacher" durchgeführt, einem ca. 50 ha großen Moorwald im NSG Federsee (Oberschwaben). Hier konnte sich seit ca. 80 Jahren eine weitgehend ungestörte Waldsukzession entwickeln. Vegetationskomplexe aus verschiedenen Pflanzengesellschaften wechseln sich kleinräumig ab. Die Orte der Probennahmen befanden sich in Bereichen, die als Vaccinio-Pinetum (Waldkiefern-Moorwald) einzustufen sind (GRÜTTNER \& WARNKEGRÜTTNER 1992). Sie zeigen eine geschlossene Kiefern-Baumschicht (Pinus sylvestris L.) von ca. 12-15m Höhe mit einer durchschnittlichen Dichte von 11,5 Bäumen $/ 100 \mathrm{~m}^{2}$. Im Unterwuchs (bis ca. $8 \mathrm{~m}$ Höhe) findet sich die Moorbirke (Betula pubescens EHRH.). Die Strauchschicht wird überwiegend von Weiden (Salix multinervis DÖLL.) gebildet. In der Krautund Moosschicht dominieren die Sphagnen. Die Dichte der Epiphyten (Hypogymnia physodes, pleurococcide Grünalgen) nimmt mit der Stammhöhe im allgemeinen schnell ab. 


\section{Fangmethode}

Sämtliche Tiere wurden mit Hilfe von Stammeklektoren erbeutet. Die selbstgebauten Modelle entsprechen in ihrer Form der Konstruktion von MÜHLENBERG (1976), besitzen aber, in Anlehnung an die Geräte von BÜCHS (1988), untere Auffangplatten mit 2 integrierten Fanggefäßen. Als Fangflüssigkeit diente 4\%ige Formollösung mit etwas Detergenz zur Verminderung der Oberflächenspannung.

Im Rahmen der durchgeführten Untersuchungen kamen Stammeklektoren erstmalig in verschiedenen Stammhöhen zum Einsatz, aber nicht - wie bisher üblich-mehrere Eklektorringe am selben Stamm, sondern immer nur eine Fangvorrichtung pro Baum. Die Fangergebnisse der einzelnen Eklektoren beeinflussen sich in diesem Fall also nicht gegenseitig. Diese Anordnung erlaubt deshalb keine eindeutige Festlegung von Bewegungsrichtungen, ermöglicht aber Vergleiche der Eklektorfaunen unterschiedlicher Stammhöhen.

Im Mai 1991 wurden 9 Eklektoren an Waldkiefern (Pinus sylvestris) montiert. An jedem der 3 Standorte wurden die Fallen in 1, 4 und $8 \mathrm{~m}$ Höhe über dem Erdboden angebracht (je 3). Der Stamm unterhalb des Eklektors war frei von Ästen, vor allem um störende "Abzweigungen" für stammbelaufende Tiere auszuschalten. Dazu wurden geeignete Stämme ausgesucht und vorhandene, durchweg abgestorbene Astreste entfernt. Im Zeitraum vom 21.5.1991 bis zum 15.10.1991 erfolgte einmal pro Woche die Leerung der Fallen. Gleichzeitig wurden Dosen, Stutzen und Eklektorinnenraum von Spinnweben gereinigt.

Zum Erklettern der Stämme diente ein Baumsteiggerät Typ BVA "Baumvelo".

\section{Klimamessungen}

MitHilfevonPICHE-Evaporimeternwurde dieEvaporation im Stammbereich gemessen. Je ein Gerät befand sich unmittelbar über jedem Eklektor auf der Nordseite des Baumes, je ein weiteres am Fuß der $1 \mathrm{~m}$-Fangbäume. Der Abstand zwischen Evaporimeterscheibe und Stamm- bzw. Bodenoberfläche betrug $4 \mathrm{~cm}$.

Die Extremwerte der Temperatur wurden mit Minima-Maxima-Thermometern nach SIX festgehalten, die ebenfalls auf der Nordseite der Stämme über den Eklektoren befestigt waren.

Beide Parameter wurden entsprechend den Fallenleerungen wöchentlich erfaßt. 
Determination

Die Artdetermination erfolgte vorwiegend nach ROBERTS (1985) und HEIMER \& NENTWIG (1991), die Nomenklatur richtet sich ebenfalls nach HEIMER \& NENTWIG (1991). Inadulte Exemplare wurden in die jeweils kleinstmögliche taxonomische Einheit eingruppiert.

\section{ERGEBNISSE}

Mikroklima

Abbildung 1 gibt die Werte der mittleren Evaporation, bezogen auf den Zeitraum zwischen der 20. und der 41 . Woche 1991, für die verschiedenen Stammhöhen wieder.

Abb. 1 Vertikalverlauf der mittleren Evaporation

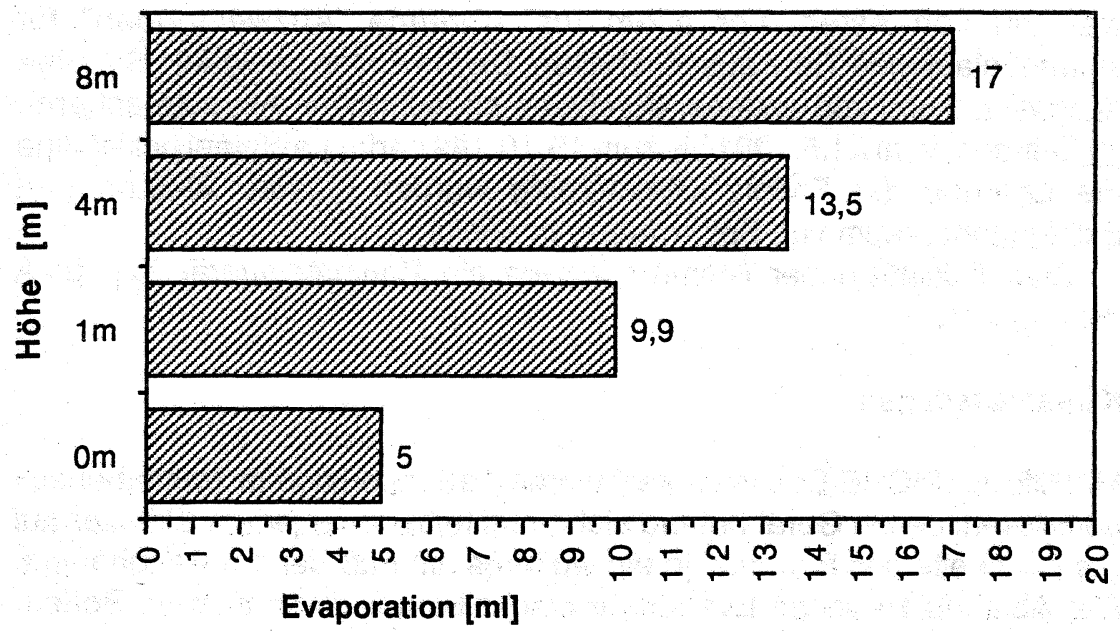

Das Temperaturprofil (Abb. 2), bezogen auf denselben Zeitraum, verdeutlicht die unterschiedlichen Verläufe von Minimal-und Maximaltemperaturen. Ausschlaggebend hierfür dürfte vor allem die in tieferen Bereichen ausgeprägtere Beschattung durch die Vegetation sein. 
Abb. 2 Vertikalverlauf der maximalen und minimalen Durchschnittstemperaturen

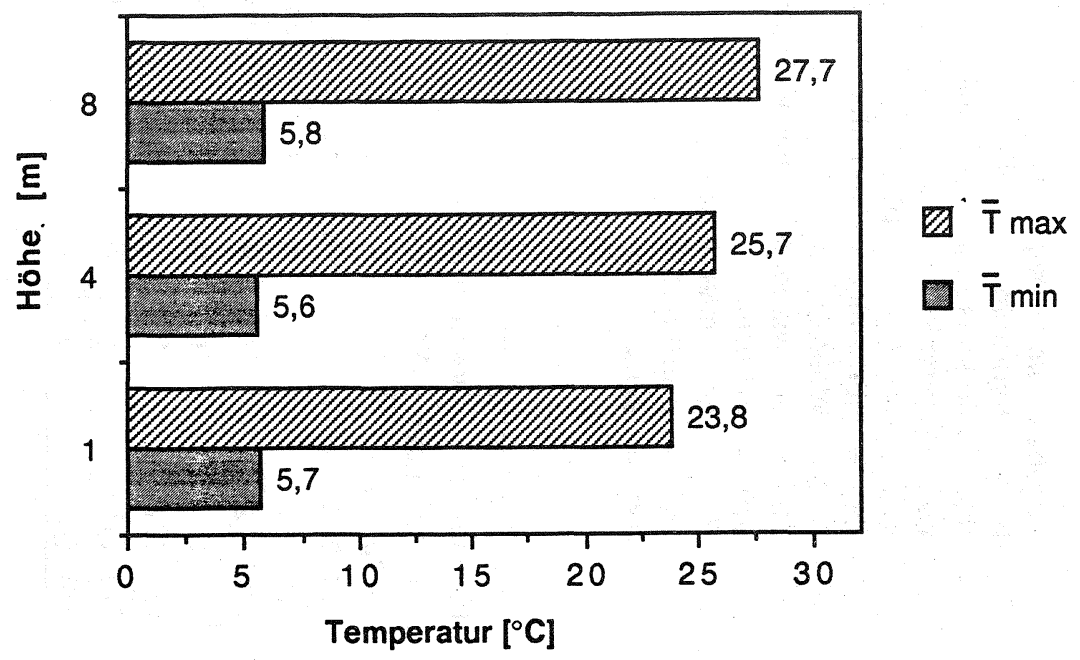

Aktivitätsdichte und Artenvielfalt

Während des Untersuchungszeitraumes wurden 3709 adulte und 3838 juvenile Spinnen aus 108 Arten bzw. 19 Familien erbeutet (Tab. 1).

Die Artenzahl nimmt mit zunehmender Stammhöhe ab: $83 \%$ wurden mit den $1 \mathrm{~m}$-Eklektoren erfaßt, die $4 \mathrm{~m}$-Ekelektoren lieferten $71 \%$ und die $8 \mathrm{~m}$ Eklektoren lediglich 62\%. 48 Arten wurden in jeder Höhe nachgewiesen, darunter befinden sich sämtliche häufigeren ( $\geq 15$ Individuen). Unter den weniger häufigen Arten und Einzelfunden tritt die weitaus größte Zahl, 21 Arten, in den $1 \mathrm{~m}$-Eklektoren auf. 9 Arten fanden sich nur in den mittleren, 8 nur in den oberen Eklektoren.

Die Gruppenaktivitätsdichte nimmt mit der Höhe zu:

$\begin{array}{cc}\text { Höhe } & \text { Individuen gesamt } \\ 8 \mathrm{~m} & 2817 \\ 4 \mathrm{~m} & 2441 \\ 1 \mathrm{~m} & 2289\end{array}$

Sofern genügend Exemplare vorlagen, wurden die Fänge in Bezug auf die Vertikalverteilung auch auf Artniveau ausgewertet, wozu alle Ergebnisse von Eklektoren gleicher Höhe addiert wurden. 
Tab. 1 Artenliste

$$
w=\text { weiblich }
$$$$
\mathrm{m}=\text { männlich }
$$

Angaben zum Ökotyp (ÖT):

h $=$ hygrobiont /-phil

(h) = überwiegend hygrophil

eu $=$ euryök

$x=$ xerobiont $/$-phil

$(x)$ = überwiegend xerophil

$w=$ Waldart (silvicol)

(w) = überwiegend / auch in Wäldern

arb $=$ arboricol

$\mathrm{R}=$ an / unter Rinde

$\mathrm{th}=$ thermophil

syn $=$ synanthrop

$a$

\begin{tabular}{|c|c|c|c|c|c|c|}
\hline \multirow[t]{2}{*}{ Familie } & \multirow[t]{2}{*}{ Gattung/Art } & Individuen & \multicolumn{3}{|c|}{ Vertikalverteilung } & \multirow[t]{2}{*}{ ÖT } \\
\hline & & $\mathrm{w} / \mathrm{m}$ (juv) & $\sum 1$ & $\sum 4$ & $\sum 8$ & \\
\hline Agelenidae & Cryphoeca silvicola (C.L.Koch 1834) & $11 / 20(6)$ & 30 & 4 & 3 & (h) \\
\hline Anyphaenidae & Anyphaena accentuata (Walckenaer 1802) & $122 / 208 \quad(197)$ & 87 & 200 & 240 & (h)w, arb,th \\
\hline \multirow[t]{6}{*}{ Araneidae } & Araneus angulatus Clerck 1757 & $1 / 1 \quad()$ & 0 & 1 & 1 & (h)w \\
\hline & Araneus diadematus Clerck 1757 & $2 / 17 \quad(2)$ & 6 & 9 & 7 & $(x)(w)$ \\
\hline & Araneus marmoreus Clerck 1757 & $1 / 3()$ & 1 & 0 & 3 & $h(w)$ \\
\hline & Araniella cucurbitina (Clerck 1757) & $0 / 2(2)$ & 1 & 1 & 2 & arb \\
\hline & Atea sturmi (Hahn 1831) & $5 / 0 \cdot(1)$ & 1 & 5 & 0 & arb \\
\hline & Gibbaranea gibbosa (Walckenaer 1802) & $1 / 0()$ & 0 & 0 & 1 & \\
\hline
\end{tabular}




\begin{tabular}{|c|c|c|c|c|c|c|}
\hline & Gibbaranea omoeda (Thorell 1870) & $0 / 2()$ & 2 & 0 & 0 & arb \\
\hline & Larinioides patagiatus (Clerck 1757) & $0 / 2()$ & 4 & 8 & 6 & \\
\hline & Nuctenea umbratica (Clerck 1757) & $1 / 15 \quad(2)$ & 0 & 0 & 2 & arb $\mathrm{R}$ \\
\hline \multirow[t]{8}{*}{ Clubionidae } & Clubiona compta C.L.Koch 1839 & $0 / 3()$ & 3 & 0 & 0 & $(x) w$ \\
\hline & Clubiona lutescens Westring 1851 & $31 / 10 \quad(2)$ & 36 & 6 & 1 & h w \\
\hline & Clubiona pallidula (Clerck 1757) & $35 / 88()$ & 31 & 38 & 58 & $(x) w$ arb \\
\hline & Clubiona reclusa O.P.-Cambridge 1863 & $0 / 1()$ & 1 & 0 & 0 & eu \\
\hline & Clubiona subsultans Thorell 1875 & $53 / 103 \quad(74)$ & 40 & 59 & 132 & $(x) w$, arb \\
\hline & Clubiona subtilis L.Koch 1866 & $0 / 3 \quad()$ & 2 & 0 & 1 & $\mathrm{~h}$ \\
\hline & Clubiona trivialis C.L.Koch 1843 & $0 / 1 \quad()$ & 0 & 1 & 1 & $(x)(w)$ \\
\hline & Clubiona sp. & $0 / 0 \quad(88)$ & 16 & 24 & 44 & \\
\hline Dictynidae & Dictyna pusilla Thorell 1856 & $17 / 30 \quad(3)$ & 8 & 22 & 20 & $x$ \\
\hline Gnaphosidae & Micaria subopaca Westring 1861 & $1 / 1(1)$ & 0 & 3 & 0 & $\operatorname{arb} \mathrm{R}$ \\
\hline Hahniidae & Hahnia pusilla C.L.Koch 1841 & $2 / 1 \quad()$ & 1 & 1 & 1 & (h)w \\
\hline \multirow[t]{9}{*}{ Linyphiidae } & Agyneta conigera (O.P.-Cambridge 1871) & $5 / 8()$ & 13 & 0 & 0 & (h)w \\
\hline & Agyneta subtilis (O.P.-Cambridge 1863) & $1 / 0 \quad()$ & 0 & 0 & 1 & $h(w)$ \\
\hline & Araeoncus humilis (Blackwall 1841) & $0 / 1 \quad()$ & 1 & 0 & 0 & (h) th \\
\hline & Bathyphantes nigrinus (Westring 1851) & $3 / 0 \quad()$ & 2 & 1 & 0 & h w \\
\hline & Bathyphantes parvulus (Westring 1851) & $1 / 00$ & 0 & 1 & 0 & eu th \\
\hline & Centromerus arcanu's (O.P.-Cambridge 1873) & $1 / 0 \quad()$ & 1 & 0 & 0 & \\
\hline & Ceratinella brevipes (Westring 1851) & $1 / 1()$ & 2 & 1 & 1 & $\mathrm{~h}$ \\
\hline & Ceratinella brevis (Wider 1834) & $4 / 0()$ & 0 & 2 & 0 & (h)w \\
\hline & Cnephalocotes obscurus (Blackwall 1834) & $0 / 1()$ & 1 & 0 & 0 & eu \\
\hline
\end{tabular}




\begin{tabular}{|c|c|c|c|c|c|c|}
\hline & Dicymbium tibiale (Blackwall 1836) & $3 / 1 \quad()$ & 2 & 2 & 0 & hw \\
\hline & Diplocephalus latifrons (O.P.-Cambridge 1863) & $3 / 0()$ & 2 & 0 & 1 & (h)w \\
\hline & Diplostyla concolor (Wider 1834) & $0 / 30$ & 1 & 3 & 0 & (h) \\
\hline & Dismodicus elevatus (C.L.Koch 1838) & $265 / 163$ & 67 & 152 & 212 & arb \\
\hline & Drapetisca socialis (Sundevall 1832) & $13 / 32(11)$ & 40 & 13 & 3 & arb R \\
\hline & Entelecara congenera (O.P.-Cambridge 1879) & $2 / 4()$ & 2 & 2 & 2 & (h)w, arb \\
\hline & Entelecara penicillata (Westring 1851) & $392 / 421 \quad()$ & 229 & 286 & 298 & arb $\mathrm{A}$ \\
\hline & Erigone atra Blackwall 1831 & $0 / 1 \quad()$ & 0 & 0 & 1 & eu \\
\hline & Erigone dentipalpis (Wider 1834) & $0 / 1 \quad()$ & 0 & 0 & 1 & eu \\
\hline & Floronia bucculenta (Clerck 1757) & $2 / 1 \quad()$ & 2 & 1 & 0 & $h(w)$ \\
\hline & Gongylidiellum latebricola (O.P.-Cambridge 1871) & $6 / 9()$ & 10 & 5 & 0 & $(x)(w)$ \\
\hline & Gongylidium rufipes (Linné 1758) & $1 / 30$ & 4 & 0 & 0 & $h(w)$ \\
\hline & Hypomma cornutum (Blackwall 1833) & $102 / 66()$ & 19 & 47 & 102 & (h), arb \\
\hline & Kaestneria dorsalis (Wider 1834) & $0 / 1 \quad()$ & 0 & 1 & 0 & $\mathrm{~h}$ \\
\hline & Lepthyphantes flavipes (Blackwall 1854) & $0 / 1 \quad()$ & 1 & 0 & 0 & $(x) w$ \\
\hline & Lepthyphantes mengei Kulczynski n1881 & $1 / 1 \quad()$ & 0 & 2 & 0 & $h(w)$ \\
\hline & Lepthyphantes minutus (Blackwall 1833) & $0 / 110$ & 8 & 1 & 2 & arb $R$ \\
\hline & Lepthyphantes obscurus (Blackwall 1841) & $4 / 6()$ & 7 & 3 & 0 & \\
\hline 5 & Linyphia hortensis Sundevall 1830 & $1 / 00$ & 1 & 0 & 0 & $(\mathrm{~h}) \mathrm{w}$ \\
\hline & Linyphia triangularis (Clerck 1757) & $20 / 27 \quad(5)$ & 42 & 8 & 3 & $e u(w)$ \\
\hline & Maso sundevalli (Westring 1851) & $4 / 00$ & 2 & 1 & 1 & $(x) w$ th \\
\hline & Meioneta innotabilis (O.P.-Cambridge 1863) & $16 / 41 \quad$ & 35 & 12 & 10 & arb $\mathrm{R}$ \\
\hline & Meioneta rurestris (C.L.Koch 1836) & $0 / 2()$ & 1 & 1 & 0 & $(x)$ \\
\hline
\end{tabular}




\begin{tabular}{|c|c|c|c|c|c|c|}
\hline & Neriene clathrata (Sundevall 1830) & $3 / 0()$ & 3 & 1 & 0 & (h)w \\
\hline & Neriene emphana (Walckenaer 1842) & $9 / 18()$ & 7 & 16 & 6 & $(h) w$ \\
\hline & Neriene montana (Clerck 1757) & $1 / 20()$ & 18 & 2 & 1 & (h)w \\
\hline & Neriene peltata (Wider 1834) & $6 / 7()$ & 7 & 3 & 3 & $(x) w$ \\
\hline & Neriene sp. & $0 / 0 \quad(21)$ & 16 & 5 & 0 & $\therefore$ \\
\hline & Oedothorax apicatus (Blackwall 1850) & $0 / 2()$ & 1 & 1 & 0 & $\mathrm{x}$ \\
\hline & Pelecopsis elongata (Wider 1834) & $21 / 4 \quad()$ & 11 & 3 & 11 & $(h) w$ \\
\hline & Pityohyphantes phrygianus (C.L.Koch 1836) & $0 / 10$ & 0 & 1 & 0 & w \\
\hline & Pocadicnemis juncea Locket \& Millidge 1953 & $0 / 1 \quad()$ & 0 & 1 & 0 & h \\
\hline & Pocadicnemis pumila (Blackwall 1841) & $2 / 30$ & 4 & 1 & 0 & eu \\
\hline & Poeciloneta globosa (Wider 1834) & $0 / 3()$ & 2 & 1 & 0 & \\
\hline & Tapinocyba pallens (O.P.-Cambridge 1872) & $74 / 4 \quad()$ & 65 & 7 & 6 & w \\
\hline & Tiso vagans (Blackwall 1834) & $1 / 0()$ & 0 & 1 & 0 & (h) th \\
\hline & Trematocephalus cristatus (Wider 1834) & $92 / 88 \quad(54)$ & 59 & 84 & 91 & arb \\
\hline & Troxochrus nasutus Schenkel 1925 & $7 / 3()$ & 8 & 0 & 1 & $w$ \\
\hline & Walckenaeria cuspidata Blackwall 1833 & $1 / 0()$ & 1 & 0 & 0 & $h(w)$ \\
\hline Liocranidae & Agroeca brunnea (Blackwall 1833) & $6 / 0()$ & 6 & 0 & 0 & $e u(w)$ \\
\hline \multirow[t]{2}{*}{ Lycosidae } & Pardosa sp. & $0 / 0(1)$ & 0 & 0 & 1 & \\
\hline & Pirata hygrophilus (Thorell 1872) & $0 / 0 \quad(121)$ & 40 & 39 & 42 & $h(w)$ \\
\hline \multirow[t]{4}{*}{ Metidae } & Metellina mengei (Blackwall 1869) & $2 / 0()$ & 2 & 0 & 0 & (h)w \\
\hline & Metellina segmentata (Clerck 1757) & $5 / 14 \quad()$ & 6 & 7 & 6 & $(h) w$ \\
\hline & Metellina sp. & $0 / 0 \quad(3)$ & 0 & 3 & 0 & \\
\hline & Zygiella atrica (C.L.Koch) & $0 / 1()$ & 0 & 0 & 1 & $\mathrm{x}, \mathrm{arb}$ \\
\hline
\end{tabular}




\begin{tabular}{|l|l|l|l|l|c|c|}
\hline Mimetidae & Ero furcata (Villers 1789) & $2 / 0(2)$ & 4 & 0 & 0 & $(\mathrm{x})(\mathrm{w})$ \\
\hline Philodromidae & Philodromus aureolus (Clerck 1757) & $16 / 10()$ & 6 & 9 & 11 & arb R \\
\hline & Philodromus cespitum (Walckenaer 1802) & $3 / 0()$ & 0 & 2 & 1 & $\mathrm{x}$ arbR \\
\hline & Philodromus collinus C.L.Koch 1835 & $22 / 29()$ & 9 & 11 & 31 & arb R \\
\hline & Philodromus dispar Walckenaer 1825 & $2 / 0(1)$ & 3 & 0 & 0 & arb, (x)w \\
\hline & Philodromus emarginatus (Schrank 1803) & $0 / 1()$ & 0 & 0 & 1 & arb \\
\hline & Philodromus fuscomarginatus (de Geer 1778) & $4 / 20(28)$ & 10 & 20 & 20 & arb R \\
\hline & Philodromus margaritatus (Clerck 1757) & $1 / 7(12)$ & 4 & 6 & 10 & arb R, th \\
\hline & Philodromus rufus (Walckenaer 1825) & $15 / 20(21)$ & 11 & 25 & 22 & $(\mathrm{x})(\mathrm{w})$ arb \\
\hline & Philodromus sp. & $0 / 0(65)$ & 8 & 26 & 31 & \\
\hline & Pisaura mirabilis (Clerck 1757) & $0 / 0(2)$ & 2 & 0 & 0 & eu \\
\hline Pisauridae & $5 / 12(5)$ & 5 & 16 & 1 & arb \\
\hline Salticidae & Dendryphantes rudis (Sundevall 1832) & $6 / 0()$ & 0 & 5 & 1 & arb R \\
\hline & Euophrys erratica (Walckenaer 1826) & $1 / 0()$ & 0 & 1 & 0 & $(\mathrm{~h})(\mathrm{w})$ \\
\hline & Neon reticulatus (Blackwall 1853) & $16 / 30()$ & 11 & 15 & 20 & $\mathrm{~h}$ arb \\
\hline & Salticus cingulatus (Panzer 1797) & $1 / 5()$ & 2 & 1 & 3 & arb R \\
\hline & Salticus zebraneus (C.L.Koch 1837) & $0 / 0(11)$ & 5 & 4 & 1 & \\
\hline & Salticus sp. & $0 / 1(1)$ & 2 & 0 & 0 & hw \\
\hline & Pachygnatha listeri Sundevall 1830 & $7 / 13(16)$ & 6 & 17 & 13 & $(\mathrm{~h}) \mathrm{w}$ \\
\hline & Tetragnatha montana Simon 1874 & $29 / 9(2)$ & 2 & 23 & 16 & euw, arb \\
\hline & Tetragnatha obtusa C.L.Koch 1837 & $1 / 8()$ & 2 & 6 & 1 & $(\mathrm{~h}) \mathrm{w}$, arb \\
\hline & Achaearanea lunata (Clerck 1757) & $1 / 4()$ & 1 & 4 & 0 & $\times(\mathrm{w})$, arb R? \\
\hline & Achaearanea simulans (Thorell) & $0 / 0(1)$ & 1 & 0 & 0 & \\
\hline
\end{tabular}




\begin{tabular}{|c|c|c|c|c|c|c|}
\hline & Enoplognatha ovata (Clerck 1757) & $10 / 27 \quad(2)$ & 24 & 10 & 5 & $(x)(w)$ \\
\hline & Robertus arundineti (O.P.-Cambridge 1871) & $1 / 0()$ & 1 & 0 & 0 & $h(w)$ \\
\hline & Steatoda bipunctata (Linné 1758) & $1 / 3(1)$ & 2 & 2 & 1 & syn, arb th \\
\hline & Theridion mystaceum L.Koch 1870 & $44 / 163 \quad(42)$ & 141 & 71 & 37 & arb $\mathrm{R}$ \\
\hline & Theridion pallens (Blackwall 1834) & $10 / 8(1)$ & 6 & 8 & 5 & $(x) w$, arb \\
\hline & Theridion pinastri L.Koch 1872 & $2 / 10()$ & 1 & 3 & 8 & $(x)$ w, arb \\
\hline & Theridion tinctum (Walckenaer 1802) & $63 / 49(44)$ & 37 & 57 & 62 & arb \\
\hline & Theridion varians Hahn 1831 & $1 / 24(1)$ & 15 & 4 & 7 & $(x) w$, arb \\
\hline \multirow[t]{5}{*}{ Thomisidae } & Coriarachne depressa (C.L.Koch 1837) & $14 / 66 \quad(52)$ & 15 & 46 & 71 & arb $\mathrm{R}$, th \\
\hline & Diaea dorsata (Fabricius 1777) & $20 / 16 \quad(10)$ & 10 & 22 & 14 & $(x) w, a r b$ \\
\hline & Xysticus audax (Schrank 1803) & $8 / 16 \quad()$ & 6 & 11 & 14 & arb \\
\hline & Xysticus lanio C.L.Koch 1835 & $0 / 1 \quad()$ & 1 & 0 & 0 & $(x) w$, arb \\
\hline & Xysticus sp. & $0 / 0 \quad(26)$ & 4 & 5 & 10 & \\
\hline Zoridae & Zora spinimana (Sundevall 1833) & $0 / 0 \quad(1)$ & 1 & 0 & 0 & eu \\
\hline \multirow[t]{9}{*}{ Jungspinnen } & Anyphaenidae & & 21 & 76 & 7 & \\
\hline & Araneidae & & 4 & 8 & 5 & \\
\hline & Clubionidae & & 69 & 29 & 24 & \\
\hline & Linyphiidae & & 289 & 302 & 394 & \\
\hline & Philodromidae & & 5 & 60 & 119 & \\
\hline & Salticidae & & 0 & 40 & 23 & \\
\hline & Theridiidae & & 43 & 18 & 65 & \\
\hline & Thomisidae & & 2 & 6 & 0 & \\
\hline & unklar & & 477 & 369 & 433 & \\
\hline
\end{tabular}


Bei 15 Arten läßt sich eine deutliche Zunahme der Aktivitätsdichte mit der Stammhöhefeststellen. Dabeihandelt es sichausschließlich um Arboricole; 6 davon gelten als obligate Rindenbewohner (WUNDERLICH 1982, PLATEN 1984). In 4 von 6 Fällen, in denen auch die Juvenilen sicher determiniert werden konnten, ergibt sich für sie die gleiche Verteilung. 8 Arten zeigen mehroderweniger deutlich eine Abnahme der Aktivitätsdichte mit zunehmender Höhe. 4 dieser Arten sind Bewohner der tieferen Strata, die anderen 4 obligate oder fakultative Rindenbewohner. Praktisch gleichverteilt sind die Zahlen bei der Wolfspinne Pirata hygrophilus, die als epigäisch gilt. Erhöhte Fangzahlen in $4 \mathrm{~m}$ Höhe (gegenüber geringeren in $1 \mathrm{~m}$ und $8 \mathrm{~m}$ ) treten bei 3 Arten auf, von denen wiederum 2 Arboricole sind (vergl. Tab. 1).

\section{Ökotypen}

Die Artenliste (Tab. 1) enthält auch Angaben zum Ökotyp (überwiegend nach PLATEN 1984, 1989, ergänzt nach TRETZEL 1952 und BLISS 1984).

$76 \%$ der erfaßten Arten, darunter praktisch alle in größeren Quantitäten erbeuteten, zählen zu den fakultativen oder obligaten Waldbewohnern. Als arboricol können 36\% der Spezies eingestuft werden. Zieht man nur die häufiger erbeuteten Arten heran, so steigt der Anteil auf $64 \%$. Obligate Rindenbewohner sind mit 15\% (bzw. 26\%) verteten.

Obwohl es sich um einen Moorstandort handelt, treten neben vielen mehr oder weniger hygrophilen Arten auch relativ zahlreich solche mit überwiegend xerophilem Charakter auf. Vergleicht man die Aktivitätsindividuendichten der Arten der Ökotypen $\mathrm{h}$ und $\mathrm{x}$ (insgesamt 23 Arten) in den unterschiedlichen Fanghöhen, so ergibt sich folgendes Bild (Abb. 3).

\section{Dominanz}

Nachdem die Jungspinnen meist nicht zur Art determiniert werden konnten, wurde für die Dominanzberechnungen auf Artniveau nur mit den Zahlen der Adulten operiert. Auf Familienniveau (Gruppendominanz) wurden die Jungspinnen, die nicht sicher einer Familie zuzuordnen waren, von der Gesamtindividuenzahl abgezogen. 
Abb. 3 Vertikalverteilung der xerophilen und hygrophilen Spinnen

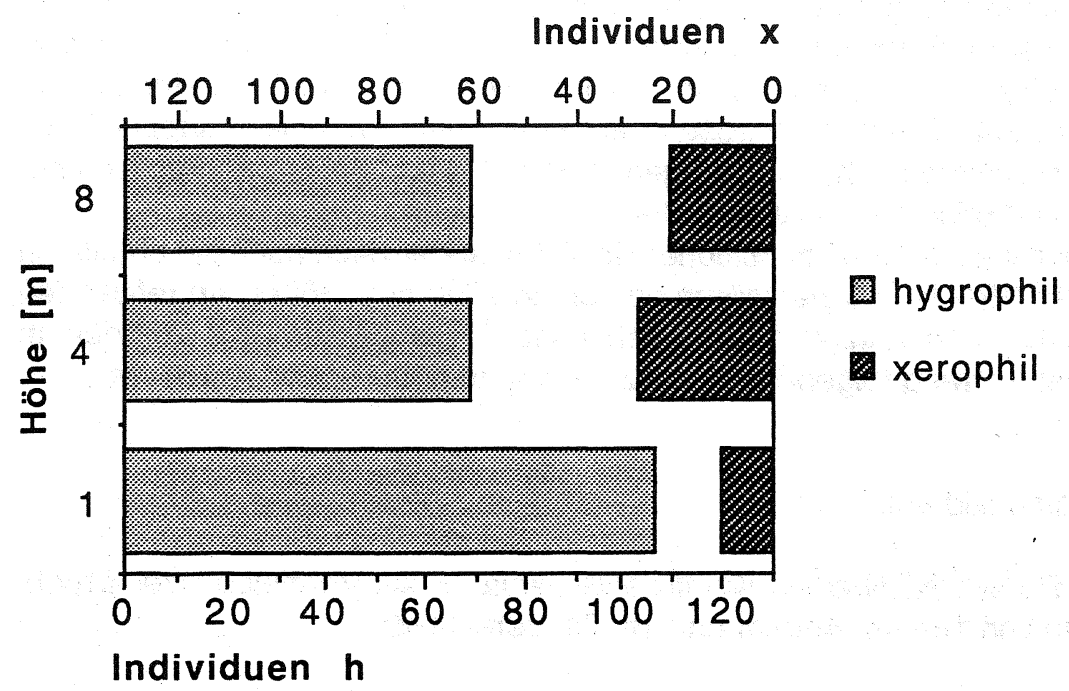

Den größten Anteil stellen die Linyphiidae mit annähernd der Hälfte der Individuen (49,8\%). Auf die Linyphiidae folgen die Theridiidae mit $10,3 \%$, die Anyphaenidae mit 10,1\%, die Clubionidae mit 9,8\%, die Philodromidae mit $7,1 \%$ sowie die Thomisidae mit $3,8 \%$. Alle weiteren Familien sind mit Werten $<3 \%$ als rezedent (ENGELMANN 1978) einzustufen.

Die bei weitem häufigste Art ist die corticole Linyphiide Entelecara penicillata mit 21,9\% der adulten Individuen. Es folgt Dismodicus elevatus mit 11,6\%. Beide Arten sind dominant. Als subdominant und damit noch zu den Hauptarten (MÜHLENBERG 1989) gehörend folgen Anyphaena accentuata (arb) mit 8,9\%, Theridion mystaceum (arbR) mit 5,6\%, Trematocephalus cristatus (arb) mit 4,9\%, Hypomma cornutum (arb) mit 4,5\%, Clubiona subsultans (arb) mit 4,2\% sowie Clubiona pallidula (arb) mit $3,3 \%$.

Auf allen drei Höhenstufen dominieren die gleichen 5 Spinnenfamilien: Linyphiidae, Theridiidae, Clubionidae, Anyphaenidae und Philodromidae. An erster Stelle stehen durchweg die Linyphiidae, die Positionen auf den Rängen 2 bis 5 dagegen wechseln von Stufe zu Stufe stark. 
Ein etwas vielfältigeres Bild ergibt sich, wenn man die Dominanzen auf Artniveau betrachtet. Den Spitzenplatz nimmt die in allen Höhenstufen dominante Entelecara penicillata ein. In $1 \mathrm{~m}$ Höhe tauchen unter den 6 häufigsten Arten neben Arboricolen auch noch zwei Vertreter tieferer Strata auf: Tapinocyba pallens als Streu- und Linyphia triangularis als Krautschichtbewohner. Dagegen spielen in 4 und $8 \mathrm{~m}$ Höhe praktisch nur noch arboricole Arten eine Rolle.

Neben den Dominanzpositionen der Arten ändern sich auch die absoluten Werte: Entelecara penicillata erreicht in allen drei Höhen ungefähr den gleichen Dominanzwert, mit wachsender Höhe werden aber von den im Dominanzrang folgenden Arten zunehmend höhere Werte erreicht.

\section{Dominanzidentität}

Die RENKONENschen Koeffizienten wurden als Vergleiche der Ergebnisse von $1 \mathrm{~m} / 4 \mathrm{~m}, 4 \mathrm{~m} / 8 \mathrm{~m}$ und $1 \mathrm{~m} / 8 \mathrm{~m}$ berechnet:

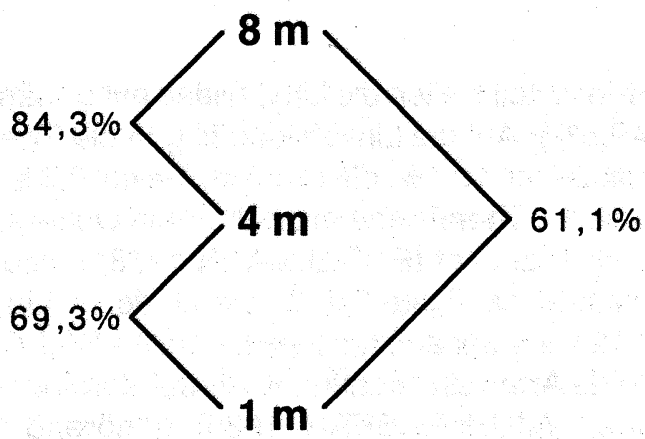

Diversität und Evenness

Die Diversität $\mathrm{H}_{\mathrm{s}}$ und die Evenness $\mathrm{E}$ wurden für die 3 Fanghöhen nach adulten Tieren aus insgesamt 103 Arten berechnet:

$\begin{array}{ccc}\text { Höhe } & \text { Hs } & \text { E } \\ 8 \mathrm{~m} & 2,80 & 0,68 \\ 4 \mathrm{~m} & 3,02 & 0,71 \\ 1 \mathrm{~m} & 3,33 & 0,76\end{array}$




\section{DISKUSSION}

Nach ALBERT (1982) werden mit Stammeklektoren Spinnen erfaßt, die

- die Bäume einmal im Jahr verlassen oder besteigen

- regelmäßig zwischen den Straten pendeln

- von den Bäumen geweht werden und sie wieder besteigen

- im Bereich der Stammeklektoren leben

- während oder nach der Kopula auf der Suche nach Partner oder Eiablageplatz auch Bäume besteigen.

Die auftretenden vertikalen Verteilungsmuster der Aktivitätsdichten sind die Folge einer bestimmten Nutzung des Stammbereiches durch die betreffenden Arten. Wichtigste Faktoren sind die Art des Nahrungserwerbes (Jäger, Lauerer, Netzbauer) sowie Präferenzen für bestimmte Stratotope, Strukturen und Biochorien. Ferner spielenverschiedene Verhaltensweisen eine Rolle: Lokalisation und Ausdehnung von Jagdrevieren, bevorzugte Orte für den Kokonbau, Agilität und Aktionsradius.

Besondere Aufmerksamkeit verdient in diesem Zusammenhang auch die Tatsache, daß der größte Teil der adulten Individuen zurKopulationszeit erfaßt wurde, die Verteilungsmuster also, besonders bei stenochronen Arten, vor allem die Aktivität des Fortpflanzungsgeschehens wiedergeben. Ein Vergleich der Muster von Männchen und Weibchen stenochroner Arten mit langlebigen Weibchen zeigt aber, etwa im Fall der häufigen Arten Hypomma cornutum und Dismodicus elevatus, kaum qualitative Unterschiede, so daß man von der Relevanz der Verteilungen in Bezug auf die ökologischen Ansprüche der Arten ausgehen kann.

Bei der Interpretation der Ergebnisse muß außerdem die Fangcharakteristik der Eklektoren Berücksichtigung finden. Die Konstruktion ist vor allem für aufbaumende Arthropoden gedacht, jedoch werden auch entlang der Stämme abbaumende erfaßt (FUNKE 1971, ELLENBERG 1973). Quantitative Angaben über die Fangeffizienz in den beiden Richtungen liegen nicht vor. Man kann aber davon ausgehen, daß aufbaumende Tiere in stärkerem Maße erfaßt werden als abbaumende. Dies bedeutet für die gewählte Eklektoranordnung, daß das Einzugsgebiet, bezogen auf die Stammoberfläche, mit der Anbringungshöhe zunimmt.

Die ausschließlich bei Arboricolen auftretende Zunahme der Aktivitätsdichte mit der Stammhöhe läßt sich in den meisten Fällen als ein Ausstrahlen der Kronenfauna in den Stammbereich auffassen. Dies gilt jedenfalls für Arten mit deutlicher Präferenz für den Kronenraum wie Anyphaena accentuata, Clubiona subsultans, Clubiona pallidula, 
Philodromus aureolus/collinus, Xysticus audax und Salticus cingulatus unter den Lauf- und Lauerjägern sowie für Hypomma cornutum, Dismodicus elevatus, Trematocephalus cristatus und Theridion tinctum unter den Netzbauern. Im Fall derRindenbewohner mit gleichem Muster, Philodromus fuscomarginatus, Coriarachne depressa und Entelecara penicillata, besteht neben einer Präferenz für höhere Stammbereiche auch die Möglichkeit, daß sich der Aktionsradius dieser Arten auf die gesamte Stammlänge erstreckt und deshalb in den $8 \mathrm{~m}$-Eklektoren mehr Individuen erfaßt wurden als in den tieferen. Das spiegelbildliche Verteilungsmuster, d.h. mit der Stammhöhe abnehmende Aktivitätsdichte, zeigen vor allem typische Bewohner der Kraut- und Streuschicht wie Tapinocyba pallens, Linyphia triangularis und Clubiona lutescens, die den Stammansatz offenbar mitbenutzen, jedoch nur selten in höhere Bereiche vorstoßen. Anders als bei den Collembolen (BRAUN 1992 bzw. i. Vorb., vergl. BAUER 1979, FUNKE 1983) zeigen sich bei den Spinnen aber keinerlei Zusammenhänge zwischen bestimmten Witterungsbedingungen und dem Vordringen epigäischer Arten in die Stammschicht. Die Stammfüße gehören offenbar zum natürlichen Aktionsraum.

Eine von der Fanghöhe unabhängige Fangquote deutet auf einen gezielten Auf- oder Abstieg in bzw. aus dem Kronenraum hin. Dieses Verteilungsmuster tritt typischerweise bei Lepidopteren- und Pentatomidenlarven auf (BRAUN 1992), aber auch bei der Lycoside Pirata hygrophilus. Alle 121 erbeuteten Individuen waren fortgeschritten juvenil bis subadult und wurden im Zeitraum zwischen Untersuchungsbeginn und Mitte Juli, besonders im Mai und Anfang Juni erfaßt. Die Bewegung geht also der Imaginalhäutung der Artmehroderwenigerunmittelbarvoran. Wie bereits ENGELHARDT (1958) vermutet, dürfte dieses Phänomen damit zusammenhängen, "daß die Lycosiden in juvenilem Zustand zu den fliegenden Spinnen gehören". Ob die Tiere aus den umliegenden Riedfluren in den Wald verdriftet wurden oder aber die Bäume als Startplätze nutzen wollten, läßt sich allerdings nicht entscheiden.

Für weitergehende, detailliertere Angaben auf Artniveau sei auf die zugrundeliegende Diplomarbeit (BRAUN 1992) verwiesen.

OCHSNER (1928) konnte für Moose zeigen, daß an Stammfüßen noch hygrophile Arten zu finden sind, während jene der Kronenregion und vor allem die Stammbewohner äußerste Trockenheit zu ertragen vermochten. Ganz entsprechend fanden PSCHORN-WALCHER \& GUNHOLD (1957) bei der Untersuchung der Kleintiersynusien in Baummoosen und -flechten am Stammfuß eine Mischfauna aus echten Bodentieren und mehr xero- 
philen Kraut- und Baumbewohnern. Die edaphischen Arten mit höheren Feuchtigkeitsansprüchen traten stammaufwärts aber bald zurück und xerophil-corticiphile beherrschten das Bild. Ebenso stell SIMON (1989) fest, daß die Rinde von Kiefern eine gewisse Attraktivität für streubewohnende Spinnen besitzt und sie den Stamm zumindest bis in eine Höhe von $2 \mathrm{~m}$ mitbenutzen. Dieses Phänomen spiegelt sich auch in der Verteilung der xerophilen und hygrophilen Spinnen wieder (Abb. 3). Für diese Arten scheint die Luftfeuchtigkeit der bestimmende Faktor zu sein, während für die Ökotypen $(h)$ und $(x)$ offenbar zunehmend andere Gegebenheiten entscheidend sind.

Faßt man die Ergebnisse der Erfassung abiotischer und trophischer Faktoren sowie die vertikale Veränderung der Spinnenfauna zusammen, solassen sich entlang der Baumstämme schematisch folgende Gradienten legen:

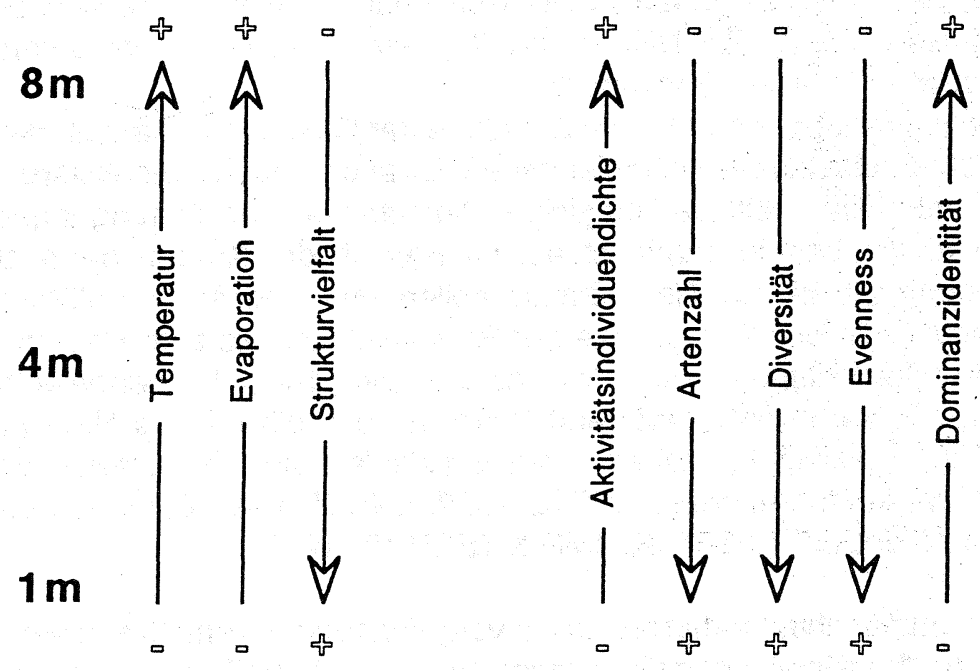

Bezüglich Artenzahl, Diversität und Evenness ergibt sich ein klares Bild, das von zwei Faktoren maßgeblich bestimmt sein dürfte: die Gradienten der abiotischen Parameter weisen die höheren Stammbereiche als deutlich extremeren Lebensraum als die tieferen aus, die Abnahme der Strukturvielfalt in gleicher Richtung kann man als zunehmende Eintönigkeit auffassen. Dem entspricht die Gestalt der Fauna: geringere Artenzahl und wenige beherrschende Arten im oberen, höhere Artenzahl und gleichmäßigere Dominanzstrukturen im unteren Stammabschnitt. Ein nicht zu 
vernachlässigender Faktor ist sicherlich auch in der deutlich abnehmenden Vermischung der Faunenelemente mit zunehmender Stammhöhe zu sehen. Zu beachten ist, daß hier keine Lebensgemeinschaften im eigentlichen Sinn, Stratocoenosen, miteinander verglichen werden. Die Fallenfänge lassen nicht unbedingt Aussagen über die Herkunft des Tieres und den Grund seines Auftretens in der Falle zu. Es handelt sich also eher um eine Art Thanatocoenose, eine Totengemeinschaft, um einen Begriff aus der Paläobiologie zu entlehnen. Am unproblematischsten erscheint mir aber der mehr deskriptive Begriff der Eklektorfauna.

Wie die RENKONENschen Zahlen zeigen, sind sich die Eklektorfaunen aus $4 \mathrm{~m}$ und $8 \mathrm{~m}$ wesentlich ähnlicher als die aus $1 \mathrm{~m}$ und $4 \mathrm{~m}$ Höhe. Geringste Ähnlichkeit besteht zwischen den Fängen aus $1 \mathrm{~m}$ und $8 \mathrm{~m}$. Auch das Dominanzgefüge bei den $1 \mathrm{~m}$-Fängen spricht dafür, daß sich hier, ähnlich wie in der Krautschicht (LUCZAK 1959), die Faunen verschiedener Straten besonders stark vermischen, denn schon in $4 \mathrm{~m}$ Höhe spielen Krautschicht- oder Streubewohner kaum mehr eine Rolle. Entsprechende Ergebnisse erhielt TURNBULL (1960) beim Vergleich von Spinnenpopulationen in einem Eichenwald.

Mit ausschlaggebend für die Zunahme der Gesamtaktivitätsdichte mit der Stammhöhe könnte im vorliegenden Fall auch eine Art Kanalisierungseffekt sein. Die Zahl der möglichen Aufenthalts- und Durchgangsorte nimmt nach obenhin zunächst $a b$, im Bereich der Kronen dann aber schlagartig wieder zu. Die Stämme stellen also eine Art Nadelöhr dar, besonders von der Obergrenze der Strauchschicht bis zum Beginn der Kronen. Dementsprechend wird das Bild hier durch Kronenbewohner geprägt, denen neben genügend Substrat auch ein reiches Nahrungsangebot zur Verfügung stehen dürfte und die deshalb in beachtenswerten Dichten vorkommen (vergl. HESSE 1939, ENGEL 1941, ENGELHARDT 1958, HÖREGOTT 1960, KLOMP \& TEERINK 1973).

Insgesamt gesehen entsprechen die vorgefundenen Verhältnisse weitgehend den "biozönotischen Grundprinzipien" von THIENEMANN: je vielfältiger die Lebensbedingungen einer Lebensstätte, um so höher die Artenzahl derzugehörigen Lebensgemeinschaft, jeeinseitiger die Lebensbedingungen eines Biotops, um so artenärmer, aber auch charakteristischer, wird die Biozönose und in um so größeren Individuenzahlen treten die einzelnen Arten auf. Faktoren sind offenbar Vielfalt der räumlichen Strukturen, Angebot an nutzbaren Ressourcen (Nahrung, Netzbau- und Unterschlupfmöglichkeiten) (vergl. SCHWERDTFEGER 1975, ALBERT 1982), mikroklimatische Bedingungen sowie Feind- oder Konkurrenzdruck. 
Dieses Prinzip gilt aber nicht nur für den Biotop als Ganzes, sondern auch für seine Habitate. So werden die höchsten Artenzahlen an Baumstämmen mit reich strukturienter Borke wie Eiche (NICOLAl 1985) oder Kiefer (PLATEN 1989) gefunden, während vor allem die glatten Stämme der Buche nur geringe Attraktivität besitzen (FUNKE 1979, NICOLAI 1985). Diewenigen Bewohner der glatten Stämme, wie Drapetisca socialis, treten dort allerdings mit hohen Abundanzen auf.

Wie die vorliegende Untersuchung zeigt, läßt sich dieses Prinzip auch auf die verschiedenen Bereiche der Stämme einer Baumart übertragen.

\section{LITERATUR}

ALBERT, R. (1982): Untersuchungen zur Struktur und Dynamik von Spinnengesellschaften verschiedener Vegetationstypen im Hoch-Solling. Diss. Univ. Bremen. $147 \mathrm{~S}$.

BAUER, T. (1979): Die Feuchtigkeit als steuernder Faktor für das Kletterverhaiten von Collembolen. - Pedobiologia 19: 165-175

BLISS, P. (1984): Untersuchungen zur Ökologie der Spinnen (Arachnida: Araneae) verschieden strukturierter Kiefernforste mit besonderer Berücksichtigung des Präferenzverhaltens derWolfspinne Pardosa lugubris (WALCKENAER1802). Diss. Univ. Halle-Wittenberg. 166S.

BRAUN, D. (1992): Ökologische Untersuchungen an Arthropoden an Kiefernstämmen unter besonderer Berücksichtigung der Araneae, Collembola und Coleoptera. Diplomarbeit Univ. Tübingen. $177 \mathrm{~S}$.

BÜCHS, W. (1988): Stamm- und Rindenzoocoenosen verschiedener Baumarten des Hartholzauenwaldes und ihr Indikatorwert für die Früherkennung von Baumschäden. Diss. Univ. Bonn. $631 \mathrm{~S}$.

ELLENBERG, H. (Hrsg) (1973): Ökosystemforschung. Springer, Heidelberg. $280 \mathrm{~S}$.

ENGEL, H. (1941): Beiträge zur Faunistik der Kiefernkronen in verschiedenen Bestandestypen. - Mitt. Forstwirtsch. u. Forstwissensch. 12: 334-361

ENGELHARDT, W. (1958): Untersuchungen über die Spinnen aus Fichtenwipfeln. - Opuscula zool. 17: 1-9

ENGELMANN, H.-D. (1978):ZurDominanzklassifizierung von Bodenarthropoden. - Pedobiologia 18: $378-380$

FUNKE, W. (1971): Food and energy turnover of leaf-eating insects and their influence on primary production. In: H. ELLENBERG (Hrsg): Integrated experimental ecology. Ecol. Stud. 2: 81-93. Springer, Berlin.

FUNKE, W. (1979): Wälder, Objekte derÖkosystemforschung: die Stammregion - Lebensraım und Durchgangszone von Arthropoden. - Jber. naturwiss. Ver. Wuppertal, 32: 45-50

FUNKE, W. (1983): Waldökosysteme in der Analyse von Struktur und Funktion - Untersuchungen an Arthropodenzönosen. - Verh. Ges. Ökol. 10: 13-26 (für 1981)

GRÜTTNER, A. \& R. WARNKE-GRÜTTNER (1992): Botanisches Gutachten für das NSG Federsee. Bericht des Projektes "Ökologische Untersuchungen zur Optimierung des Schutzes der Flora und Fauna im Naturschutzgebiet Federsee" (1989-1991) (Forschungsvorhaben U22 - 89.04), botanischer Teil, Tübingen. $143 \mathrm{~S}$. 
HEIMER, S. \& W. NENTWIG (Hrsg) (1991): Spinnen Mitteleuropas. 1. Aufl., Parey, Berlin u. Hamburg. $543 \mathrm{~S}$.

HESSE, E. (1939): Untersuchungen an einer Kollektion Wipfelspinnen. - Sber. Ges. naturf. Freunde Berlin 39 (8-10): 350-363

HÖREGOTT, H. (1960): Untersuchungen über die qualitative und quantitative Zusammensetzung der Arthropodenfauna in Kiefernkronen. - Beitr. Ent. 10: 891-916

KLOMP, H. \& B.J. TEERINK (1973): The density of the invertebrate summer fauna on the crowns of pine trees, Pinus sylvestris, in the central part of the Netherlands. - Beitr. Ent. 23: $325-340$

LUCZAK, J. (1959): The community of spiders of the ground flora of pine forest. - Ekologia Polska, Ser. A, 7: 285-315

LUCZAK, J. (1963): Differences in the structure of communities of web spiders in one type of environment (young pine forest). - Ekologia Polska, Ser. A, 11: 159-221

LUCZAK, J. (1966): The distribution of wandering spiders in different layers of the environment as a result of interspecies competition. - Ekologia Polska, Ser. A, 14: 233-244

MÜHLENBERG, M. (1976): Freilandökologie. 1. Aufl., Quelle \& Meyer, Heidelberg u. Wiesbaden, 214 S.

MÜHLENBERG, M. (1989): Freilandökologie. 2. Aufl., Quelle \& Meyer, Heidelberg u. Wiesbaden, $430 \mathrm{~S}$.

NICOLAl, V. (1985): Die ökologische Bedeutung verschiedener Rindentypen bei Bäumen. Diss. Univ. Marburg. $198 \mathrm{~S}$.

OCHSNER, F. (1928): Studien über die Epiphytenvegetation der Schweiz. - Jb. St. Gallener Naturw. Ges. 1927/28

PLATEN, R. (1984): Ökologie, Faunistik und Gefährdungssituation der Spinnen und Weberknechte in Berlin (West) mit dem Vorschlag einer roten Liste. - Zool. Beitr. 28: 445-487

PLATEN, R. (1989): Struktur der Spinnen- und Laufkäferfauna (Arach.: Araneida, Col.: Carabidae) anthropogen beeinflußter Moorstandorte in Berlin (West); taxonomische, räumliche und zeitliche Aspekte. Diss. TU Berlin. $470 \mathrm{~S}$.

PSCHORN-WALCHER, H. \& P. GUNHOLD (1951): Zur Kenntnis der Tiergemeinschaft in Moos- und Flechtenrasen an Park- und Waldbäumen. - Z. Morph. Ökol. Tiere 46: $342-354$

ROBERTS, M.J. (1985): The spiders of Great Britain and Ireland. Vol. 1-2.1. Aufl., Harley Books, Colchester. $204 \mathrm{~S}$.

SCHEIDLER, M. (1990) Influence of habitat structure and vegetation architecture on spiders. - Zool. Anz. 225 (5/6): 333-340

SCHWERDTFEGER, F. (1975): Ökologie der Tiere: Synökologie. Bd. 3. 1.Aufl., Parey, Hamburg U. Berlin. $451 \mathrm{~S}$.

SIMON, U. (1989): Die Spinnenzönose der Kiefernrinde. Diplomarbeit FU Berlin. $138 \mathrm{~S}$.

TRETZEL, E. (1952): Zur Ökologie der Spinnen (Araneae). Autökologie der Arten im Raum von Erlangen. - Sber. phys.-med. Soc. Erlangen 75: 36-131

TURNBULL, A.L. (1960): The spider population of a stand of oak (Quercus robur L) in Wytham Wood, Berks., England. - Canad. Entomol. 92: 110-124

VITÉ, J.P. (1953): Untersuchungen über die ökologische und forstliche Bedeutung der Spinnen im Walde. $-Z$. angew. Ent. 34: 313-334

WUNDERLICH, J. (1982): Mitteleuropäische Spinnen (Araneae) der Baumrinde, - Z. ang. Ent. 94: 9-21

\section{Daniel Braun, Käsenbachstr. 10, D-W-7400 Tübingen}

